\title{
BIRADS 4 breast lesions: comparison of contrast-enhanced spectral mammography and contrast-enhanced MRI
}

\author{
Rabab Yasin ${ }^{1 *}$ and Enas Abd El Ghany ${ }^{2}$
}

\begin{abstract}
Background: Breast cancer is the most common cancer in women worldwide. It is responsible for about $23 \%$ of cancer in females in both developed and developing countries [1]. We aimed to assess the accuracy of contrastenhanced spectral mammography (CESM) versus contrast-enhanced breast MRI in the evaluation of BIRADS 4 breast lesions.

Results: Fifty patients were included in this study; there were 28 malignant cases and 22 benign cases; all cases were proved by histopathological result either by core biopsy or excision biopsy. CESM was found to have less sensitivity (94.1\%) than MRI (100\%) but CESM has higher specificity (100\%) than MRI (95.5\%). The accuracy of CESM was $96.4 \%$, while the accuracy of MRI was $98.2 \%$ with no statistical significance ( $P$ value 0.827 ).

Conclusion: CESM can be used as a sensitive diagnostic tool in the detection and staging of breast cancer with higher specificity and less sensitivity as compared to contrast enhanced breast MRI.
\end{abstract}

Keywords: BIRADS, Contrast-enhanced spectral mammography, Contrast-enhanced MRI, Breast cancer

\section{Background}

Breast cancer is the most common cancer in women worldwide. It is responsible for about $23 \%$ of cancer in females in both developed and developing countries [1]. In the last few years, the incidence and the mortality rate of breast cancer have increased about $20 \%$ \& $14 \%$ respectively [2].

The most important goal is to early diagnose breast cancer accurately and in a cost-effective way in every woman, regardless of ages, races, economic levels, risk levels, and geographic settings [2].

For so long that mammography was the only breast imaging examination that reduced breast cancer mortality, with a population-based sensitivity of $75 \%$ to $80 \%$ [3]. Mammography can detect breast cancer with variable sensitivity ranging from 63 to $98 \%$, but its sensitivity is decreased to $30-48 \%$ in the case of dense breasts [4].

Screen-film mammography is almost entirely replaced by full-field digital mammography (FFDM). Although

\footnotetext{
* Correspondence: Rabab_yasin@outlook.com; Rababyasin123@gmail.com ${ }^{1}$ Department of Radiology, Faculty of Medicine, Menofia University, Al Minufiyah, Egypt

Full list of author information is available at the end of the article
}

FFDM has the advantages of higher imaging quality and higher contrast resolution with faster image processing as compared to screen-film mammography, both FFDM and screen-film mammography have the same sensitivity with about half of the cases can go undetected [5].

Contrast-enhanced MRI has been used for a long time as a gold standard method for the diagnosis of breast cancer, depending upon the new angiogenesis of the lesions. But, it has many limitations as low specificity, high cost, long duration of examination time, and limited availability [6-8].

Contrast-enhanced spectral mammogram (CESM) is considered now a relatively new imaging modality which can provide both anatomic and functional information of the breast lesion similar to MRI [8].

CESM like contrast-enhanced magnetic resonance imaging (CE-MRI) is used to detect angiogenesis of the lesion in the mammography suite. It includes high- and low-energy images during a single compression after contrast injection [9].

The advantage of CESM over MRI is that it is less expensive and easier to perform with shorter examination time. Also, the higher sensitivity of MRI is plagued by 
numerous false-positive foci of enhancement [10]. Also, CESM can replace MRI in case of patient contraindicating for MRI as patients with pacemakers, aneurysm clips or metal implants, or severe claustrophobia [9].

Our study aimed to assess the accuracy of CESM in comparison to contrast-enhanced breast MRI in the evaluation of BIRADS 4 breast lesions.

\section{Methods}

Our study was a prospective study done in the period between January 2017 and May 2018 on 50 patients. The patients' age was ranged from 33 to 83 years with the mean age 52 years.

All patients had previously undergone mammography (MG) and ultrasound and had a breast lesion of BIRADS 4. We considered the histopathological results as the reference standard.

CESM was done first for all patients, followed by MRI after 7 days after CESM. MRI examination was done between the 5th and the 12th day after the start of the menstrual cycle in pre-menopausal women.

Our exclusion criteria were the following:

- Pregnancy

- History of allergic reaction to iodinated contrast agent

- Renal insufficiency

- Other contraindications to MRI include pacemaker, claustrophobia, metal prosthesis, or aneurysmal clips

All patients were subjected to physical examination and full history taking, including medical and drug history. Full explanations of the procedures were done for all patients, including the associated risks and contraindications.

A written consent was taken from all patients prior to the study to be included in our study.

CESM and MRI images were evaluated by two experienced radiologists with 15 years of interpretation experience in breast imaging, including mammography, CESM, and breast MRI.

\section{CESM technique}

CESM was done using a GE mammogram "GE Senographe $^{\text {mix }}$ Essential with SenoBright" upgrade."

Patient positioning and performance of contrastenhanced digital mammography were the same as conventional mammography.

The procedure was explained to the patient when a written consent is signed. Renal function (RF) is checked, and an IV line is secured.

Further, $1.5 \mathrm{~cm}^{3} / \mathrm{kg} 320 \mathrm{mg} \%$ Visipaque is injected IV using an electronic injector "Imaexon automated injector" after warming the contrast.
We started imaging the breast without a suspected lesion then the breast with the suspected lesion to increase the uptake of contrast in the breast of interest. Mediolateral oblique (MLO) view was done first, followed after 1 min by craniocaudal (CC) view. In each view, two image acquisition were included: a high-energy $(45-49 \mathrm{kVp})$ and a low-energy $(26-30 \mathrm{kVp})$, with the ranges of $\mathrm{kVp}$ settings depending on the thickness and density of the breast. Time separation between low- and high-energy images were typically within $1 \mathrm{~s}$ and of maximum time separation of $3 \mathrm{~s}$.

Imaging in four views was done from minute 2 to minute 6 for both breasts using a stop watch built-in in the mammogram. The procedure is done in the attendance of the radiologist to ensure patient safety and check the images. The cannula is then removed, and post injection instructions given to the patient. No adverse reaction was seen in any of our patients.

\section{Image analysis}

A subtraction image for each low- and high-energy pair was done automatically, to get the maximum conspicuity of iodinated contrast agent uptake. Subtracted CESM images are then analyzed in terms of lesion enhancement and given a score from 1 to 4 starting from negative, faint, moderate, and intense enhancement.

\section{MRI technique}

All MRI was performed on a 1.5-T system (Magnetom Aera; Siemens, Erlangen, Germany). We examined these women in prone position with their breasts fixed in a dedicated four-channel phased-array bilateral breast coil. The breast coil enabled imaging of both breasts in one image for comparison of both sides.

In premenopausal women, our study was done in the second week of the menstrual cycle in order to minimize the effect of hormonal changes on the contrast uptake of the breast.

The patients were instructed to stay still as much as possible to avoid misregistration artifacts.

Placement of an intravenous catheter was done before positioning the patient on the MR table. The contrast agent was given by a power injector. The injection of gadolinium was done with a dose of $0.1 \mathrm{mmol} / \mathrm{kg}$ (or 0.2 $\mathrm{mL} / \mathrm{kg}$ ) and a flow rate of $1-2 \mathrm{~mL} / \mathrm{s}$ flushed by $20 \mathrm{~mL}$ of physiological saline and six sequential contrast-enhanced images were acquired at every $1 \mathrm{~min}$.

We performed the following MRI sequences with parameters; slice thickness $4 \mathrm{~mm}$, FOV $350 \mathrm{~mm}$, and matrix $512 \times 512$ :

- Axial turbo inversion recovery magnitude (TIRM) with TR $7700 \mathrm{~ms}$, TE $74 \mathrm{~ms}$. 
- Axial T2 fast spin-echo (FSE) with TR 6160 ms, TE $76 \mathrm{~ms}$.

- Axial T1 fast spin-echo (FSE) with TR 415 ms, TE $4.6 \mathrm{~ms}$

- Diffusion-weighted imaging (DWI) was done TR $8200 \mathrm{~ms}$, TR $85 \mathrm{~ms}$ in the axial plane bilaterally with $b$ values of 0,500 , and $1000 \mathrm{~s} / \mathrm{mm}^{2}$.

- ADC map was systematically performed.

- For dynamic study, we acquired one pre- and six post-contrast sequences. Intravenous contrast injection of $0.1 \mathrm{mmol} / \mathrm{kg}$ of body weight of gadobutrol (Gadovist; Bayer-Schering, Berlin, Germany) at a rate $2 \mathrm{~mL} / \mathrm{s}$ using automatic MR-compatible power injector. Then performing serial dynamic images acquired six times in the axial plane with threedimensional transverse fast, low angle shot T1weighted sequence (TR/TE, 5.27/2.39 ms, FOV 260 $\mathrm{mm}$, matrix, $512 \times 512$; section thickness $1.6 \mathrm{~mm}$ ).

- Then post-processing subtraction of the precontrast images from the post-contrast images with fat suppression, multi-planar reconstruction (MPR) and maximum intensity projections (MIP).

- Bilateral sagittal T1 FSE post-contrast administration.

- Delayed axial T1 post-contrast imaging $10 \mathrm{~min}$ after contrast injection.

All patients were subjected to histopathological assessment either core or excisional biopsy, it was considered the gold standard reference in the study.

\section{Results}

Fifty patients were included in this study; all of them had a breast lesion of BIRADS 4 on conventional mammography and ultrasound examination.

The patients' age was ranged from 33 to 83 years with the mean age 52 years. In our study, there were 28 malignant cases and 22 benign cases; all cases were proved by histopathological result either by core biopsy or excision biopsy. Table 1 and chart 1 showed the histopathological results in our cases.

Table 2 showed the criteria of breast lesions as regarding the size, margin, contrast uptake, degree of enhancement, and the number of lesions on CESM and MRI.

The enhancement pattern of lesions on CESM were classified in four grades (Table 3); no enhancement, faint (Fig. 1), moderate (Fig. 2), and intense enhancement (Fig. 3). While the enhancement pattern of lesions on MRI were classified as regarding dynamic enhancement curve (Table 4).

There were four cases with no contrast uptake on CESM; three cases proven to be an intraductal papilloma and one case of fibrocystic disease. While only one case
Table 1 Histopathological results in our cases

\begin{tabular}{ll}
\hline Histopathological diagnosis & Number of cases \\
\hline IDC & $18(36 \%)$ \\
DCIS with non-mass enhancement & $3(6 \%)$ \\
ILC & $4(8 \%)$ \\
Malignant papillary cystic carcinoma & $2(4 \%)$ \\
Metastasis & $1(2 \%)$ \\
Papilloma & $4(8 \%)$ \\
Epitheliosis & $1(2 \%)$ \\
Granulomatous mastitis & $2(4 \%)$ \\
Fibrocystic disease & $3(6 \%)$ \\
Organized hematoma & $1(2 \%)$ \\
Sclerosing adenosis & $2(4 \%)$ \\
Fat necrosis & $4(8 \%)$ \\
Radial scar & $2(4 \%)$ \\
Atypical ductal hyperplasia & $3(6 \%)$ \\
Total & $50(100 \%)$
\end{tabular}

IDC invasive ductal carcinoma, DCIS ductal carcinoma in situ, ILC invasive lobular carcinoma

on MRI did not show any contrast uptake and it was proven as fibrocystic disease.

There were seven malignant cases with multiple enhancing lesions seen on MRI (Table 2); three cases of invasive ductal carcinoma (IDC) (Fig. 4), two cases of invasive lobular carcinoma (ILC), and one case of intrapapillary cystic carcinoma (Fig. 5); the last case was a false positive due to parenchymal enhancement. While CESM shows multiple enhancing lesions in five cases, the two missed cases were not detected on CESM due to small size of the lesion less than $5 \mathrm{~mm}$; one of them was a small satellite lesion while the other one was a false positive case seen on MRI due to parenchymal enhancement.

There were six cases of non-mass enhancement detected on CESM; two cases ductal carcinoma in situ (DCIS) with non-mass enhancement, two cases of IDC with DCIS, one case of fibrocystic disease, and one case of ILC (Table 2).

While non-mass enhancement was detected in seven cases on MRI; three cases of DCIS with non-mass enhancement, two cases of IDC with DCIS, one case of fibrocystic disease, and one case ILC (Table 2).

CESM missed one case with DCIS with non-mass enhancement (Fig. 6) and one satellite lesions less than $5 \mathrm{~mm}$.

For malignant lesions, we have 28 cases; six cases of them had more than one lesion and one false positive. So, we have 34 malignant lesions and 22 benign lesions which considered to be true negatives for malignancy.

\section{Statistical method}

Data were expressed as both number and percentage of the categorized data (qualitative variable). 
Table 2 The criteria of lesions on CESM and MRI

\begin{tabular}{|c|c|c|c|}
\hline Characteristic of mass & CESM & MRI & $P$ value \\
\hline Mean size & $20.7 \pm 1.3 \mathrm{~mm}$ & $20.2 \pm 1.6 \mathrm{~mm}$ & 0.086 \\
\hline \multicolumn{4}{|l|}{ Contrast uptake } \\
\hline - Positive & $46(92 \%)$ & $49(98 \%)$ & \multirow[t]{2}{*}{0.168} \\
\hline - Negative & $4(8 \%)$ & $1(2 \%)$ & \\
\hline \multicolumn{4}{|l|}{ Degree of mass enhancement } \\
\hline - Faint & $10(21.7 \%)$ & $12(26.1 \%)$ & \multirow[t]{3}{*}{0.899} \\
\hline - Moderate & $11(23.9 \%)$ & $10(21.7 \%)$ & \\
\hline • Intense & $25(54.3 \%)$ & $27(58.7 \%)$ & \\
\hline \multicolumn{4}{|l|}{ Margin of mass } \\
\hline - Well defined & $4(8.7 \%)$ & $7(15.2 \%)$ & \multirow[t]{2}{*}{0.394} \\
\hline - Irregular & $42(91.3 \%)$ & $42(91.3 \%)$ & \\
\hline The smallest size of detected lesion & $5 \pm 0.9 \mathrm{~mm}$ & $3 \pm 0.6 \mathrm{~mm}$ & $<0.001$ \\
\hline \multicolumn{4}{|l|}{ Number of lesions } \\
\hline - One lesion & $41(89.1 \%)$ & $42(91.3 \%)$ & \multirow[t]{2}{*}{0.616} \\
\hline - More than one lesion & $5(10.9 \%)$ & $7(15.2 \%)$ & \\
\hline Non mass enhancement & $6(13.0 \%)$ & $7(15.2 \%)$ & 0.860 \\
\hline
\end{tabular}

\section{Discussion}

For the detection and staging of breast cancer, MRI is considered the gold standard method. It has its disadvantage of high cost, long examination time, limited availability, and low specificity due to false positive results [6].

CESM is a newly used diagnostic tool in breast imaging with the advantage of low cost, shorter examination time, and high specificity as compared to MRI breast.

As compared to MRI, now CESM is considered a relatively new imaging modality which gives both anatomic and functional information of breast lesions similar to MRI. CESM has a lower cost and shorter duration examination than MRI [7]. Its sensitivity is comparable to MRI in detecting breast cancer [8].

Also, low-energy images of CESM (similar to digital mammography) are able to detect micro-calcifications, speculated lesion, and architectural distortion which may be missed on subtracted CESM and MRI if not enhancing [10].

In MRI, background enhancement posed a critical problem that affects the degree of accuracy of the study if not done during the proper time of the cycle (i.e., second week). Background enhancement caused no degradation to the accuracy of detection or analysis of lesions in CE mammography. Thus, CE mammography was superior to MRI in lesion detection and analysis in patients with extensive background enhancement. Also, CE mammography could be done anytime during the cycle unlike MRI so no time delay when using CE mammography [11].

On the other hand, MRI was superior to CE mammography in enhancement kinematics. On MRI, lesion enhancement was analyzed based on their enhancement pattern and kinetics with an enhancement curve plotted over time interval and the lesion enhancement was decided either progressive, a plateau or wash-out where wash-out is the worst and most suspicious form. Also, MRI was superior to CE mammography in assessing the $\mathrm{ADC}$ value of the lesions where lesions with low ADC values. While CE mammography only assessed lesion enhancement in terms of their enhancement pattern, hence less confidence in lesion characterization compared to MRI [10].

Table 3 The pattern of enhancement of lesions on CESM

\begin{tabular}{lll}
\hline Enhancement pattern & Number of cases & Histopathological result \\
\hline No enhancement & 4 & Benign lesions \\
Faint & 10 & Benign lesions \\
Moderate & 11 & 7 benign lesions and 3 malignant lesions \\
Intense & 25 & All were malignant except one case of granulomatous mastitis \\
\hline
\end{tabular}




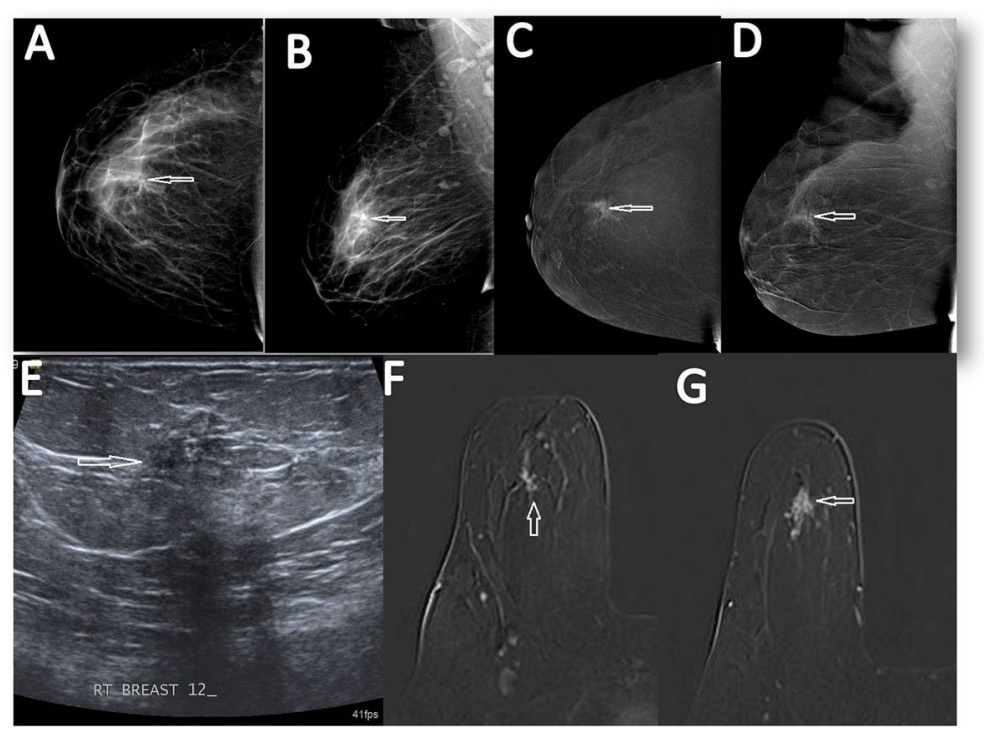

Fig. 1 Female patient 55 years old, she was complaining of left breast mass. On ultrasound (a), there was a hypoechoic mass at 9 o'clock with slight an irregular margin (arrow). On T1 MRI (b), the lesion appears hypointense with irregular outlines (arrow). On TIRM MRI (c), the lesion shows hyperintense signal with peripheral thick enhancement after IV contrast (d) with dynamic curve type I (arrows). On CESM (e, f), the lesion was hardly seen as faint marginal enhancement (arrows). A biopsy was done and histopathologically proven as fat necrosis

In our study, we did a comparative study between CESM and MRI for the ability to detect and characterize breast lesion of BIRADS 4.

We found that CESM has less sensitivity (94.1\%) than MRI (100\%) but CESM has higher specificity (100\%) than MRI (95.5\%). The accuracy of CESM was $96.4 \%$, while the accuracy of MRI was $98.2 \%$ (Table 5) with no statistical significance ( $P$ value 0.827 ).

Li et al. 2017 found that CESM has similar sensitivity than BMRI in breast cancer detection, with higher positive predictive value (PPV) and less background enhancement. They stated that CESM is associated with
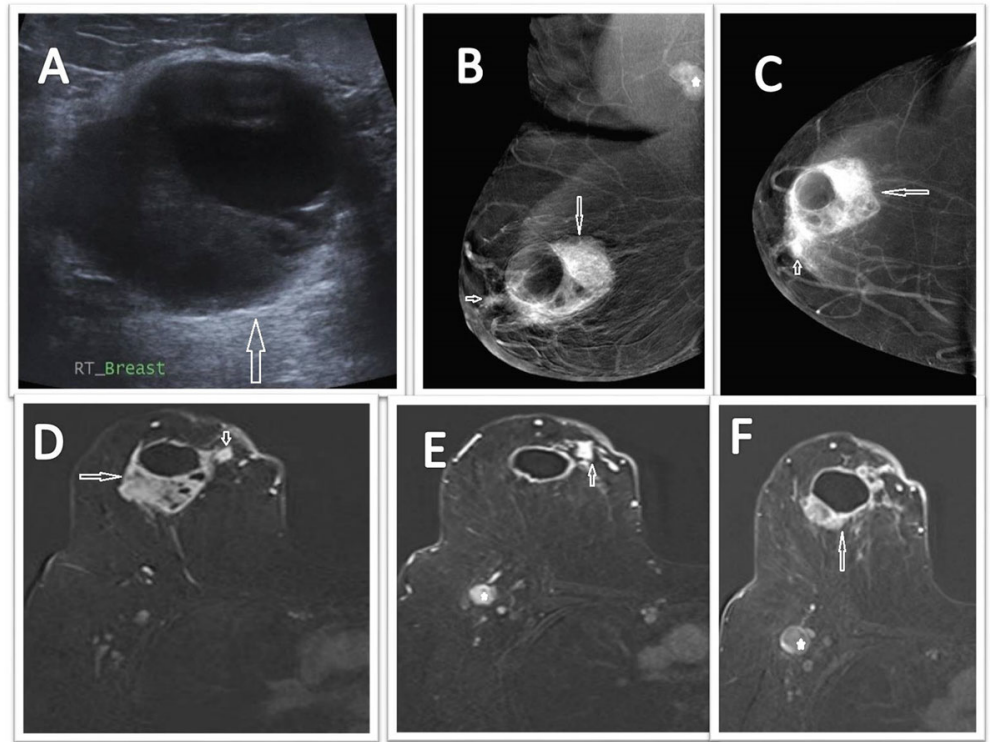

Fig. 2 Female patient 48 years old. She came with routine check-up mammography. There was an irregular speculated lesion with central lucency seen on mammography (arrows in $\mathbf{a}, \mathbf{b}$ ). An ultrasound (e), the lesion appears as an irregular, ill-defined hypoechoic lesion with posterior shadowing at 12 o'clock (arrow). On CESM (c, d), it shows moderate enhancement (arrows). On MRI with IV contrast (f, $\mathbf{g}$ ), it appears as an irregular speculated enhancing lesion with dynamic curve type I (arrows). A biopsy was done and histopathologically proven as sclerosing adenosis 

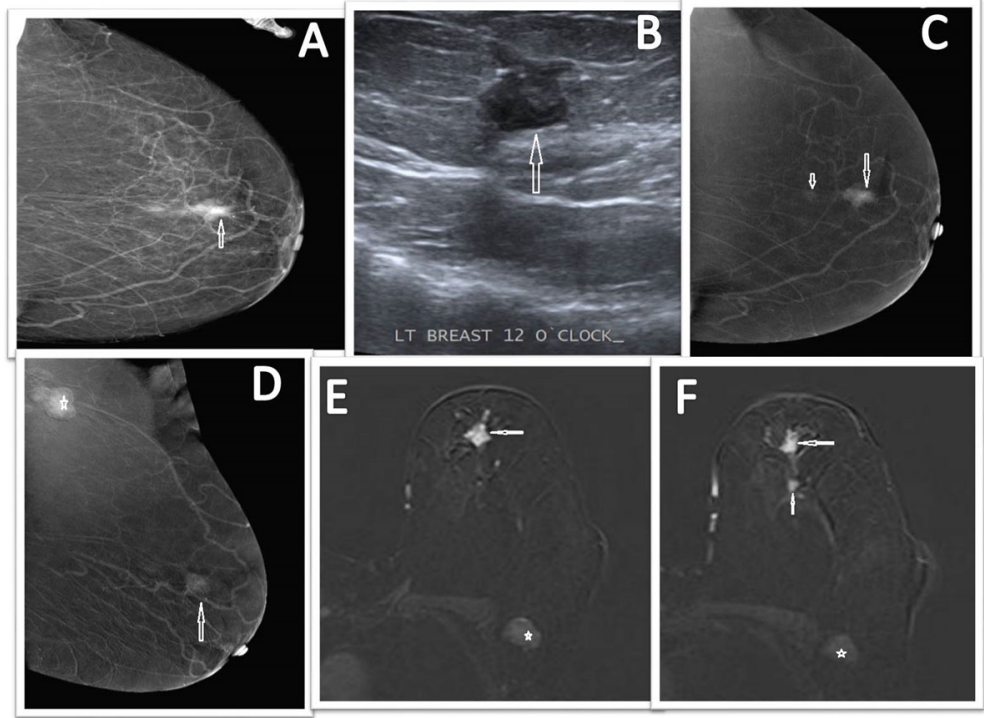

Fig. 3 Female patient 47 years old complaining of right breast mass. On conventional mammography $(\mathbf{a}, \mathbf{b})$, there was a segmental irregular lesion at the upper outer quadrant (arrows). On CESM (c, d), the lesion showed intense enhancement (arrows). It appeared as an irregular hypoechoic mass at the 10 o'clock with posterior acoustic enhancement on ultrasound (e) (arrows). No pathologically enlarged axially LNS. On CE-MRI (f, $\mathbf{g}$ ), the lesion shows heterogenous segmental enhancement extending to the nipple with dynamic curve type III (arrows). An ultrasound guided core biopsy was done and histopathologically proven as granulomatous mastitis

significantly shorter exam time thus a more accessible alternative to BMRI and has the potential to play an important tool in breast cancer detection and staging [12].

Elfiky et al. 2018 did a comparative study between CESM and BMRI; they concluded that contrastenhanced spectral mammography (CESM) showed slightly lower sensitivity (88.89\%) and overall accuracy (86.67\%) than BMRI (96.30\% and $90 \%$ respectively). But the specificity was higher in CESM (66.67\%) than that of BMRI (33.33\%). They concluded that in spite of the lower sensitivity of the CESM compared to MRI, the CESM appeared to be a suitable, easy, more comfortable, low cost, and fast alternative to MRI in early detection of breast cancer recurrence specially for patients with contraindications to MRI [13].

In a study done by Liao et al. 2017, CESM and BMRI were consistent on morphology and equal sensitivity for detection of breast cancer lesions. They stated that CESM has less enhancement intensity than BMRI and higher PPV (reflecting a higher specificity) than BMRI [14].

Table 4 The pattern of enhancement of lesions on MRI

\begin{tabular}{lll}
\hline $\begin{array}{l}\text { Type of MRI } \\
\text { curve }\end{array}$ & $\begin{array}{l}\text { Number of } \\
\text { cases }\end{array}$ & Histopathological results \\
\hline $\begin{array}{l}\text { No } \\
\text { enhancement }\end{array}$ & 1 & Benign lesion \\
Type I curve & 12 & Benign lesions \\
Type II curve & 10 & 8 benign and 2 malignant lesions (DCIS) \\
Type III curve & 27 & $\begin{array}{l}26 \text { malignant lesions and 1benign case } \\
\text { (granulomatous mastitis) }\end{array}$ \\
\hline
\end{tabular}

This was in agreement with Jochelson et al. 2013 who performed a prospective study which concluded that CESM has a lower sensitivity and higher specificity when comparison was made to BMRI [11]. This was supported by another study done by Roth et al. 2017; they found that CESM may have a higher specificity than BRMI, reflected by a higher PPV. The PPV on CESM is higher than PPV on BMRI. The difference is not statistically significant [10]. These results may be due to small sample size, which contributed to non-significance of PPV. Also, higher specificity of CESM as result of lower breast parenchymal enhancement on CESM than MRI [10].

Also, Yousef et al. 2018 and James 2018 were in agreement with the findings of Houssami et al. $[6,15]$; they found that CESM have lower sensitivity and higher specificity of breast cancer. The limited specificity of breast MRI, leading to additional work-up and biopsies with false-positive results [16].

A study on CESM was done by Jong et al. 2003; they show the ability of CESM to detect breast malignant lesions especially in dense breast [17]. Another extended study by Diekmann et al. 2011 on contrast-enhanced digital mammography (CEDM) found that CEDM had high sensitivity and specificity for detecting breast lesions [18].

While Dromain et al. 2009 compared CESM to MRI for breast lesions. They found that MRI enhancement is more accurate than CESM for differentiating malignant from and benign lesions. They concluded that absence of contrast enhancement on CESM did not exclude 

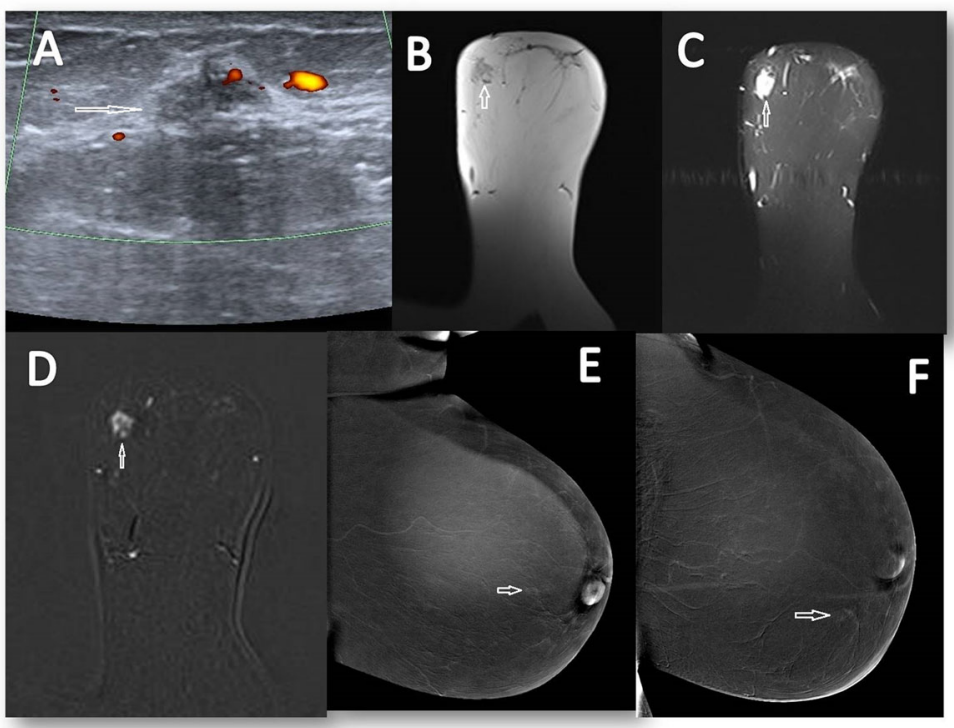

Fig. 4 Female patient 71 years old. She was presented with left breast mass. On mammography (a), slight irregular lesion is seen at 12 o'clock (arrow). On ultrasound (b), the lesion appears as a fairly defined hypoechoic lesion with a tongue-like projections (arrows). On CESM (c, d), it appears irregular with moderate enhancement (long arrow) and with enhancing axillary LNS (asterisk) on MLO view. A small enhancing nodule is seen deep to the lesion on CC view (small arrow on c), but it was not seen on MLO view. On MRI, the lesion is irregular, enhancing (long arrow) with enhancement curve type III and with a ductal linear enhancement extending posteriorly to small enhancing satellite lesion (small arrow). An enlarged pathological rounded axillary LNS (asterisk). A biopsy was done and histopathologically proven as invasive ductal carcinoma with DCIS

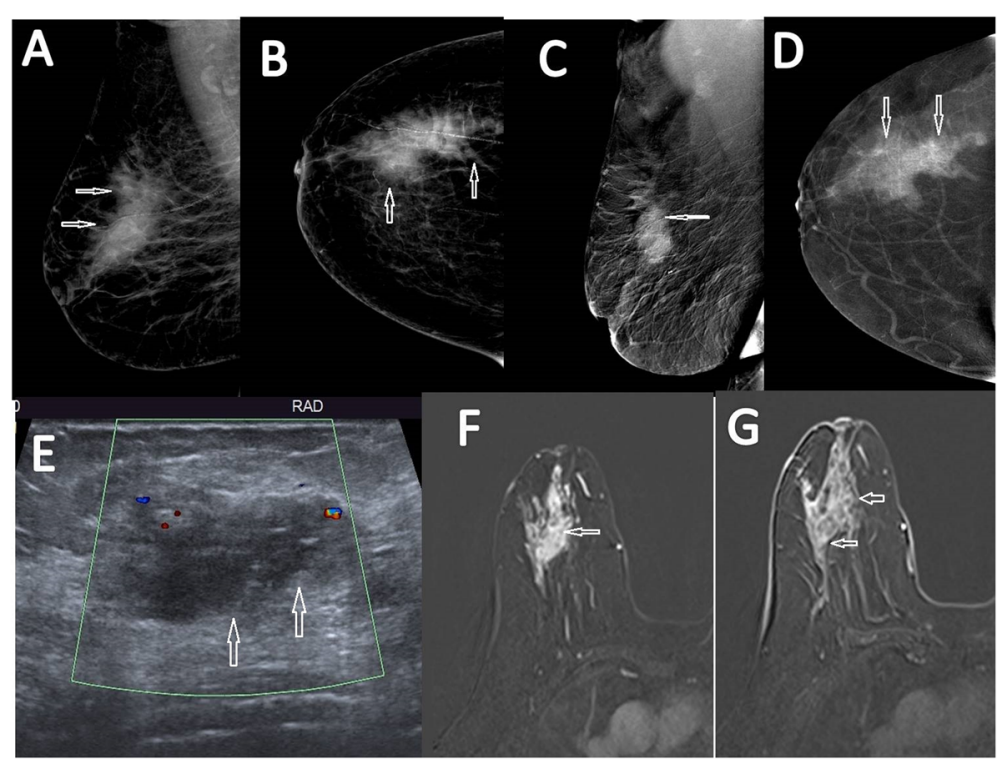

Fig. 5 Female patient 54 years old, she was presented with right breast mass. On ultrasound (a), there was a complex well-defined cystic lesion with soft tissue component at 12 o'clock (arrow), which shows flow on the color Doppler study. On CESM (b, c), the lesion shows intense enhancement of the solid component (arrows) with enlarged right axillary LNS (asterisk). A small enhancing satellite lesion is seen anterior to the lesion (short arrow). On MRI with IV contrast (e, f), the lesion appears complex cystic with enhanced large soft tissue component of dynamic curve type II and III (arrows). The satellite lesion is seen anterior and medial to the lesion (small arrow) with thickening enhancing overlying skin and enlarged pathological rounded axillary LNS (asterisk). A biopsy was done and histopathologically proven as intraductal papillary carcinoma 


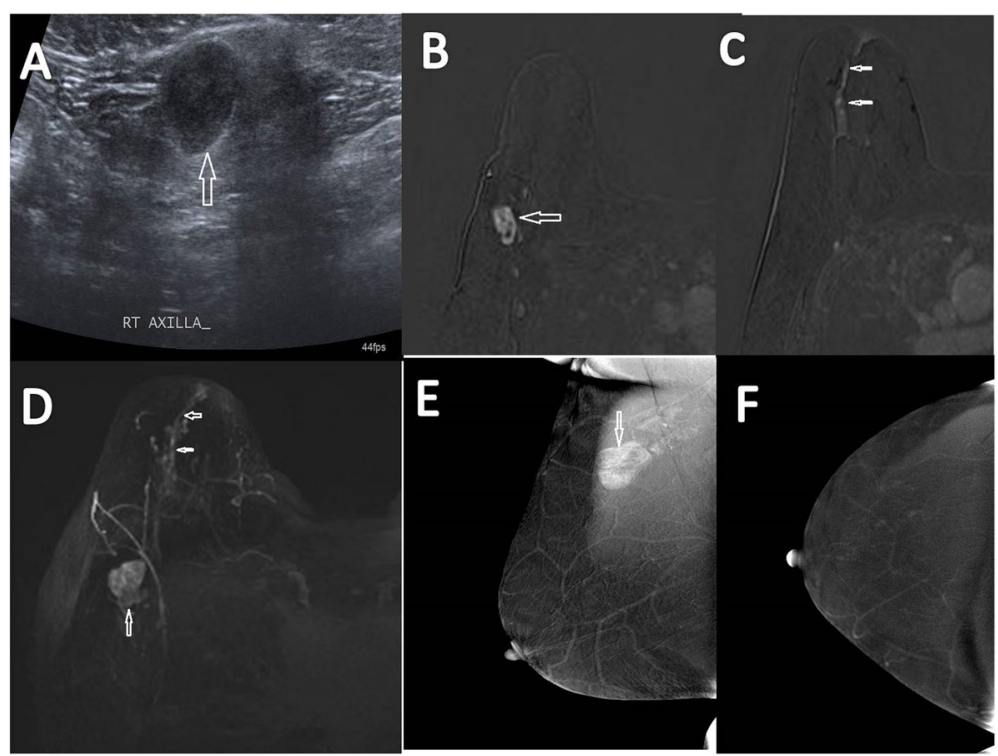

Fig. 6 Female patient 58 year old presented with right axillary swelling. On ultrasound (a), there was enlarged axillary lymph nodes with the loss of their normal fatty hilum and with a rounded appearance indicating pathological LNS (arrows), no lesion could be seen in breast in ultrasound or mammography. On MRI TIRM sequence (b), an enlarged right axillary LNS were seen (long arrow). On CE-MRI (c), there was evidence of intraductal linear enhancement directed towards the nipple (small arrows). On MIP MRI (d), the enlarged axillary LNS and ductal enhancement was clearly seen (arrows). On CESM (e, f), right axillary LNs was enhancing (arrow) while the ductal enhancement could not be seen. On histopathological correlation it was proven as DCIS with metastatic axillary LNS

malignancy. Unlike MRI non-enhancement, which was an exclusion criterion for invasive malignant tumors [19].

In our study, the mean size of the lesions on CESM was $20.7 \pm 1.3 \mathrm{~mm}$ and it was $20.2 \pm 1.6 \mathrm{~mm}$ on MRI with no statistical significance ( $P$ value 0.086$)$. So CESM was as good as MRI for evaluation of tumor size.

This was in agreement with Lobbes et al 2015 who compared CESM versus MRI for the assessment of the size of the breast tumor; they found that CESM is good as an imaging tool for measuring the size of the tumor

Table 5 Represented the sensitivity, specificity, accuracy, PPV and NPV

\begin{tabular}{llll}
\hline & CESM & MRI & $P$ \\
\hline TP & 32 & 34 & 0.827 \\
FP & 0 & 1 & \\
FN & 2 & 0 & \\
TN & 22 & 21 & \\
Sensitivity & $94.1 \%$ & $100 \%$ & \\
Specificity & $100 \%$ & $95.5 \%$ & \\
Accuracy & $96.4 \%$ & $98.2 \%$ & \\
PPV & $100 \%$ & $97.1 \%$ & \\
NPP & $91.7 \%$ & $100 \%$ & \\
\hline
\end{tabular}

CESM contrast-enhanced spectral mammography, MRI magnetic resonance imaging, $T P$ true positive, $F P$ false positive, $F N$ false negative, $T N$ true negative, $P P V$ positive predictive value, NPP negative predictive value and MRI did not improve the quality of the tumor size evaluation [20].

While Roth et al. 2017 found that the lesion size was larger on BMRI as compared to CESM with $(P<0.05)[10]$.

Łuczyńska et al. 2015 also did a comparative study between CESM and MRI; they found that the size of the lesion detected on CESM and breast MRI were the same but were larger than histopathological size with statistical significance $(P<0.01)$. The sizes of the lesions were overestimated by $1.7 \mathrm{~mm}$ on CESM and by $1.8 \mathrm{~mm}$ on MRI [21].

As regards the criteria of lesions including degree of enhancement and margin of lesions, there was no statistical significance between CESM and MRI with $P$ value of 0.899 and 0.394 respectively (Table 2 ).

In our study, the smallest enhancing lesion detected on CESM was $5 \pm 0.9 \mathrm{~mm}$ while the smallest detected size on MRI was $3 \pm 0.6 \mathrm{~mm}$ with high statistical significance $(P$ value $>0.001)$. The smallest size of the lesion was less on MRI than CESM as a result of two lesions smaller than $5 \mathrm{~mm}$ seen on MRI and not detected on CESM. One of them was a small satellite lesion in IDC while the second one was a false positive lesion on MRI due to parenchymal enhancement.

For the accuracy of CESM to detect multifocal or multicentric breast lesions. We found that CESM was able to detect multifocal or multicentric lesions in five of six cases with no statistical significance between CESM\&MRI for detection of multifocal lesions ( $P$ value 0.616$)$. 
This was in agreement with Fallenberg et al. 2014 who found that CESM can detect the number and extension of multifocal/multicentric lesion accurately [22]. Also, Dromain et al. 2009 found the important role of CESM to diagnose exact extensions of breast cancer [19].

In another study done by Dromain et al. 2011, CESM detected multifocal lesion in 23 cases versus 16 and 15 lesions detected by mammography and US respectively [23].

In our study, CESM could not detect intraductal papilloma in three cases of a total of four cases. This was in agreement with a study done by Mokhtar 2014 on CESM and MRI; they found that CESM and MRI are similar to the assessment of the local extent of disease, the size of the lesion, and multifocal or multicentric involvement. But they found that CESM was not the optimal study for some benign lesions such as intraductal papilloma or small fibroadenomas or radial scars [24].

In the case of non-mass enhancement such as in ductal carcinoma in situ, mammary adenosis, or fibrocystic disease, the detection is more difficult than mass lesions due to lack of defined margin [25].

CESM could detect non-mass enhancement in six cases, while it missed only in one case of DCIS without IDC (Fig. 6). MRI detected all cases of non-mass enhancement with no statistical significance between CESM and MRI, $P$ value 0.860 .

Lewin et al. 2003 stated that CESM has limited role in ductal carcinoma in situ as it was weakly enhancing on CESM [26].

Łuczyńska et al. (2015) did a comparative study between CESM and MRI. They found that CESM is valuable in the diagnosis and assessment of breast cancer. Their results were $100 \%$ of cancers on CESM, while being $93 \%$ positive on breast MRI [21].

However, several limitations affect temporal CEDM; there is a paucity of published research on CESM in diagnosing DCIS, non-mass enhancement, and intraductal papilloma for comparison with MR breast. More researches using large sample sizes are needed for more definitive assessment of CESM specificity.

\section{Conclusion}

We concluded that CESM is a sensitive diagnostic tool for breast cancer with higher specificity and less sensitivity as compared to contrast-enhanced breast MRI. CESM has shorter examination time, thus a more accessible alternative to MRI, and has the potential to be an important diagnostic tool in breast cancer detection and staging.

\section{Abbreviations}

BI-RADS: Breast Imaging-Reporting and Data System; CC: Craniocaudal; CEDM: Contrast-enhanced digital mammography; CE-MRI: Contrast-enhanced magnetic resonance imaging; CESM: Contrast-enhanced spectral mammography; DCIS: Ductal carcinoma in situ; DWI: Diffusion-weighted imaging; FFDM: Full field digital mammography; FN: False negative; FP: False positive; FSE: Fast spin echo; IDC: Invasive ductal carcinoma; ILC: Invasive lobular carcinoma; MG: Mammography; MIP: Maximum intensity projections; MLO: Mediolateral oblique; MPR: Multi-planar reconstruction.; NPP: Negative predictive value; PPV: Positive predictive value; RF: Renal function; TIRM: Turbo inversion recovery magnitude; TN: True negative; TP: True positive

\section{Acknowledgements}

We would like to thank all people who helped us in this work including the histopathology doctors, surgeons and technicians.

\section{Authors' contributions}

Equal sharing between authors as regarding writing of the manuscript, the collection and analysis of data, and revising the final manuscript. Both authors have read and approved the manuscript.

\section{Funding}

Self-funding.

Availability of data and materials

All data and material are available.

\section{Ethics approval and consent to participate}

The study protocol was approved by the local Ethics Committee. All study procedures were performed in accordance with the ethical standards laid down in the 1964 Declaration of Helsinki and its later amendments. No available ethics committee's reference number. A written consent was taken from all patients prior to the study to be included in our study.

\section{Consent for publication}

A written consent was taken from all patients prior to the study for publication.

\section{Competing interests}

The authors declare that they have no competing interests.

\section{Author details}

${ }^{1}$ Department of Radiology, Faculty of Medicine, Menofia University, Al Minufiyah, Egypt. ${ }^{2}$ Department of Radiodiagnosis, Faculty of Medicine, Ain Shams University, Cairo, Egypt.

Received: 30 August 2019 Accepted: 6 September 2019

Published online: 18 September 2019

\section{References}

1. Helal M, Abu Samra M, Ibraheem MA et al (2017) Accuracy of CESM versus conventional mammography and ultrasound in evaluation of Breast imaging-reporting and data system BI-RADS 3 and 4 breast lesions with pathological correlation. Egypt J Radiol Nucl Med 48:741-750

2. Siegel R, Naishadham D, Jemal A (2013) Cancer statistics, 2013. Cancer J Clin 63(1):11-30

3. Jochelson M Advanced imaging techniques for the detection of breast cancer. Am Soc Clin Oncol Educ Book 2012:65-69

4. Kolb TM, Lichy J, Newhouse JH (2002) Comparison of the performance of screening mammography, physical examination, and breast US and evaluation of factors that influence them: an analysis of 27,825 patient evaluations. Radiology 225:165-175

5. Pisano ED, Gatsonis C, Hendrick E et al (2005) Diagnostic performance of digital versus film mammography for breast-cancer screening. N Engl J Med 353:1773-1783

6. Houssami N, Ciatto S, Macaskill P et al (2008) Accuracy and surgical impact of magnetic resonance imaging in breast cancer staging: systematic review and meta-analysis in detection of multifocal and multicentric cancer. J Clin Oncol 26:3248-3258

7. Roganovic D, Djilas D, Vujnovic S et al (2015) Breast MRI, digital mammography and breast tomosynthesis: comparison of three methods for early detection of breast cancer. Bosn J Basic Med Sci 15:64-68

8. Nekhlyudov L, Kiarsis K, Elmore JG. MRI of the breast: does the internet accurately report its beneficial uses and limitations? Breast J 2009; 15: 189-93. 
9. Luczyn E, Heinze-Paluchowska S, Dyczek S et al (2014) Contrast-enhanced spectral mammography: comparison with conventional mammography and histopathology in 152 women. Korean J Radiol 15(6):689-696

10. Roth LR, Germaineb P, Renc S et al (2017) Contrast-enhanced spectral mammography (CESM) versus breast magnetic resonance imaging (MRI): A retrospective comparison in 66 breast lesions. and Interventional Imaging 98:113-123

11. Jochelson MS, Dershaw DD, Sung JS et al (2013) Bilateral contrast-enhanced dual-energy digital mammography: feasibility and comparison with conventional digital mammography and MR imaging in women with known breast carcinoma. Radiology 266(3):743-751

12. Li L, Roth R, Germaine P et al (2017) Contrast-enhanced spectral mammography (CESM) versus breast magnetic resonance imaging (MRI): A retrospective comparison in 66 breast lesions. Diagn Interv Imaging:113-123

13. Elfiky SM, Elsaid NA, Azeb EA et al (2018) Comparison between the role of contrast enhanced mammography and dynamic contrast enhanced MRI in assessment of breast cancer recurrence. Egypt J Hosp Med 73(1):5875-5885

14. Liao L, Li L, Germaine P et al (2017) contrast-enhanced spectral mammography (CESM) compared with breast MRI for breast cancer detection. J Clin Oncol:11069-11069

15. James J.J \& Tennant S.L: Contrast-enhanced spectral mammography (CESM). Clin Radiol August 2018 Volume 73, Issue 8, Pages 715-723.

16. Yousef $\mathrm{A}$, Khatera $\mathrm{H}$, Jameelb L. Contrast-enhanced spectral mammography versus magnetic resonance imaging in the assessment of breast masses. Benha Med J, January-April 2018 Vol. 35 No. 1.

17. Jong R, Yaffe M, Skarpathiotakis M et al (2003) Contrast-enhanced digital mammography: initial clinical experience. Radiology 228:842-850

18. Diekmann F, Freyer M, Diekmann S et al (2011) Evaluation of contrastenhanced digital mammography. EUR Radiol 78:112-121

19. Dromain C, Balleyguier C, Adlera G, Garbay JR, Delaloge S (2009) Contrastenhanced digital mammography. Eur Radiol 69:34-42

20. Lobbes MB, Lalji UC, Nelemans PJ et al (2015) The quality of tumor size assessment by contrast-enhanced spectral mammography and the benefit of additional breast MRI. J Cancer 6

21. Łuczyńska E, Heinze-Paluchowska S, Hendrick E et al (2015) Comparison between breast MRI and contrast-enhanced spectral mammography. Med Sci Monit 21:1358-1367

22. Fallenberg EM, Dromain C, Diekmann F, Engelken F, Krohn M, Singh JM et al (2014) Contrast- enhanced spectral mammography versus MRl: initial results in the detection of breast cancer and assessment of tumor size. Eur Radiol 24:256-64. -198

23. Dromain C, Thibault F, Muller S, Rimareix F, Delaloge S, Tardivon A et al (2011) Dual energy contrast-enhanced digital mammography: initial clinical results. Eur Radiol 21:565-574

24. Mokhtar O, Mahmoud S (2014) Can contrast enhanced mammography solve the problem of dense breast lesions? Egypt J Radiol Nucl Med 45: 1043-1052

25. Newell D, Nie K, Chen JH, Hsu CC, Yu HJ, Nalcioglu O et al (2009) Selection of diagnostic features to differentiate between malignant and benign lesions that presented as mass lesions and non-mass type enhancement on breast MRI. Proc intl soc mag reson med:658

26. Lewin JM, Isaacs PK, Virginia V, Larke Fred J (2003) Dual-energy contrastenhanced subtraction mammography: feasibility. Radiology 229:261-268

\section{Publisher's Note}

Springer Nature remains neutral with regard to jurisdictional claims in published maps and institutional affiliations.

\section{Submit your manuscript to a SpringerOpen ${ }^{\circ}$ journal and benefit from:}

- Convenient online submission

- Rigorous peer review

- Open access: articles freely available online

High visibility within the field

- Retaining the copyright to your article

Submit your next manuscript at $\boldsymbol{\nabla}$ springeropen.com 